\title{
The optical effect of thermal expansion over excessed injection molded lens arrays in modern LED outdoor luminaires
}

\author{
Viktor Zsellér \\ GE Lighting Hungary Kft. \\ 1044 Budapest, Váci Út 77. \\ Viktor.Zseller@ge.com
}

\author{
Dr. Krisztián Samu \\ Budapest University of Technology and Economist \\ Department of Mechatronics, Optics and Engineering \\ Informatics \\ 1111 Budapest, Bertalan Lajos u. 4-6. \\ samuk@mogi.bme.hu
}

\begin{abstract}
For the rapid development observable in solid state lighting technology, both in favor of the better lighting performance achievable and the advantage in upkeep, changing the conventional installations to LED luminaires is often considerable in the modern roadway lighting practice. By these modernization investments the sales volume is usually high for lower additional expenses and a quicker RoI; thus the replacement products are generally cost sensitive. As the global market price of Aluminum increases on a long term trend, while the cost of the task efficient luminous power became very desirable with Light Emitting Diode; a high level of integration and compactness is demanded by such luminaire designs. This approach raises complex technical challenges, as the thermal expansion difference between the lens array and the Insulated Metal Substrate (IMS) Printed Circuit Board can lead to major deviation in the light distribution over the rated ambient temperature range. Having a robust design is a key aspect in providing a reliable, safe lighting design for any application of a global product.
\end{abstract}

Keywords-LED; outdoor; roadway; lighting; refractive optics; injection molding, thermal expansion

\section{INTRODUCTION}

This paper intends to introduce the technical challenges faced by the development of an outdoor roadway luminaire from the aspects of optical to thermal compatibility and its contribution to a robust design in terms of adequate light distribution provided under various environmental circumstances.

In order to maintain a high level of resistance against the destructive effects that an outdoor lighting fixture is exposed, the housing is usually made of die-cast Aluminum and the secondary lens has to be tempered glass. (Some specific markets do accept deviation from the traditional material specifications for a lower product cost, but this results worse durability and decreased thermal performance.) As the semiconductor light sources dissipate around half of the input power thermally, it is essential to understand the way of the heat flow in the system towards these usually die-cast Aluminum heat sinks.

This research published in this paper was found by the TÁMOP program of the Hungarian Government.
As the mounting of the SMT (Surface Mounting Technology) power LED light sources would be extremely complicated on the luminaire housing, these are soldered to a PCB (Printed Circuit Board), that is then fastened on the thermal dissipating surface of the top housing for an adequate heat conduction. The PCBA (Printed Circuit Board Assembly) is either FR4 type or IMS. The latter is the acronym of Insulated Metal Substrate and the technology was used initially as a special solution for solving issues of IC based power amplifiers thermal management. The main concept of this technology is that there is a thick 5052 or 6061 Aluminum sheet metal which has a laminated dielectric layer on one or both surfaces. This is covered with a copper layer that is the top surface of the PCB and electrical components are mounted upon it. It has a great advantage of better heat dissipation from the junction of the light emitting diode die. The traditional FR4 type boards provide a large thermal resistance between the chip and the ambient. In some rare cases if a $\mathrm{CoB}$ (Chip on Board) LED is used in an outdoor luminaire, it can be mounted directly to the heat sink, but in this scenario the optical control capabilities are limited and it can result a poor applicability, while electric insulation is yet another challenging factor.

The main reason of the importance of thermal management in LED fixtures is Lumen Maintenance, which is the ratio of initial and actual light output at a given time. The warmer the solder point temperature of the LED is, the quicker it depreciates its light output. The same applies usually to optics as well; both reflective coatings and refractive lens degrade more as a result of elevated temperature. The excessive range of temperature differences observable in the system lead to the complex mechanical problem of thermal compatibility.

The optical unit is either reflective or refractive in an LED roadway lighting fixture. Reflective systems can utilize special mirrors or TIR (Total Internal Reflection) technology. Refractive optical systems contain one or more special freeform lens that guide the lambertian light distribution source of the LED to a suitable form for a specific application. The latter two methods can lower the direct material cost of the product, while it demands much tighter tolerances and requires a more detailed evaluation of robustness of the design 
${ }^{[1]}$. This paper covers some aspects to consider while designing such complex fine mechanical assemblies.

\section{COMPONENT COMPATIBILITY}

\section{A. Environmental conditions}

When evaluating the robustness of the optical light engine, it is important to identify the operation circumstances and ranges claimed suitable for a given fixture, including the standards specified for roadway lighting and outdoor lighting.

The operative $T_{A}$ ambient temperature range of a LED lighting luminaire in the EMEA region is usually:

$$
-40{ }^{\circ} \mathrm{C}<\mathrm{T}_{\mathrm{A}}<50{ }^{\circ} \mathrm{C}
$$

The minimum temperature defined is generally higher by South Europe, Middle East and Turkish tenders, the maximum rated temperature required for Nordic countries may be considered lower. For a general product with sales representation over the whole region the range has to cover both extremes.

For the heat convection type thermal flow, it is always considered, that there is no forced elevation of this kind of dissipation. This is represented by the third party validations as the measurements are always performed in isolated chambers, thus considering the worst case application scenario.

It can be applied as a rule of thumb, that the temperature of any given component in the system is scaling offset wise with the $\mathrm{T}_{\mathrm{A}}$ ambient temperature. The rated parameters of a product are usually claimed for $\mathrm{T}_{\mathrm{A} 0}=25^{\circ} \mathrm{C}$ unless otherwise stated. Thus the temperature of a component based on its rated temperature at $25^{\circ} \mathrm{C} \mathrm{T}_{\mathrm{A} 0}$ is:

$$
T_{C l}=T_{C O}+\left(T_{A l}-T_{A 0}\right)
$$

This formula indicates, that the temperature of the LED board PCBA and the temperature of the optics can vary on a range of $90{ }^{\circ} \mathrm{C}$, and thus the different amount of thermal expansion causes the detuning of the optical system, that may result in failing the lighting scenarios.

\section{B. Component properties}

In order to evaluate the boundaries of the design, the thermal expansion of the three main component; namely the Polycarbonate refractive optics, the PCBA and the housing was measured considering multiple materials. The evaluation of the PCBA includes both FR4 and IMS products.

When investigating the relative position of the components to each other, it is important to understand, that for component cost reasons, one LED board always consists multiple LEDs and the refractive lens are also molded in an array. These two parts are locked together in one point and usually two additional hinges are needed to prevent translation and rotation.
The one fix point where the lens array is locked to the board is usually a boss on the lens substrate that is wielded from the clear side of the IMS.

\section{Component properties}

The following diagrams show the relative thermal expansion of these components. The key takeaway is, that the optics suffer a much bigger expansion and it causes the shifting of the light sources relative position, especially at the side positions. Note, that in the case of PCBs, there is a separation of longitudinal and transversal directions that is due to FR4 lamination technologies.
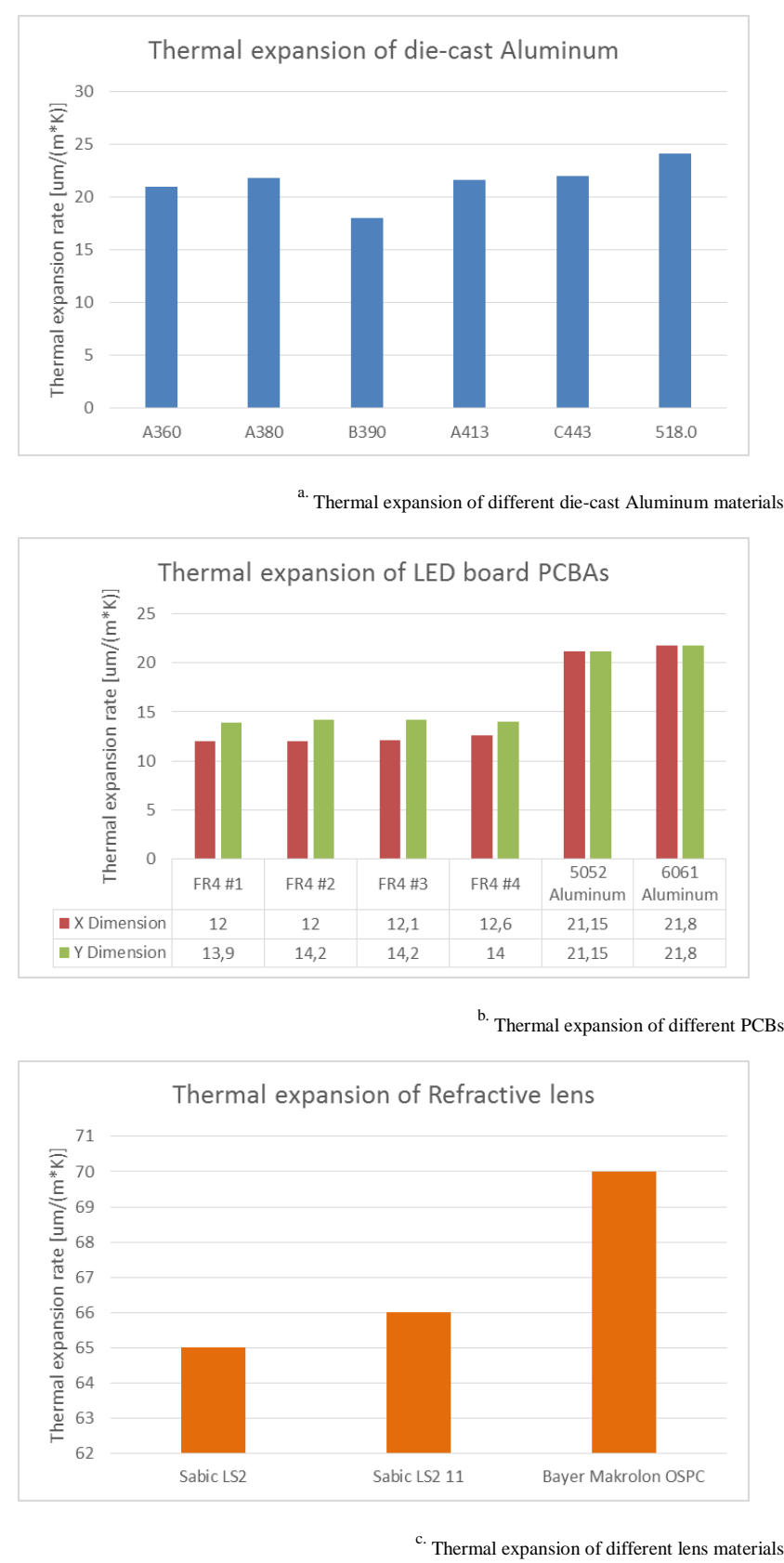

At this point is is clear to see, that the larger the excession of the optical light engine is, the bigger the deviation from the 
rated locations becomes. This causes internal stress between the housing and the PCBA, especally if FR4 is used, and additionally this effect can modify the light distribution of the luminaire.

\section{GENERAL LIGHTING REQUIREMENTS}

\section{A. Illuminance}

Illuminance is the total luminous flux incident on a surface unit per area and thus it expresses, how much the incident light illuminates the surface. For a general lighting design there can be a requirement for the minimum value of measurable Illuminance on the pavement surface, on task areas and on specified vertical surfaces that are meant to quantify facial recognition. Besides the minimum values, often the average requirement is specified as well.

\section{B. Luminance}

Luminance is the luminous intensity per unit area of light traveling in a given direction from a surface describing the amount of light observable from a particular area towards a given solid angle. Understanding the behavior of this parameter is essential, as Luminance changes dramatically by minor alternation of the system level light distribution of a roadway lighting luminaire.

For Roadway calculations there is a predefined observer in the center of each lane positioned $60 \mathrm{~m}$ apart the first luminaire of a calculation area to calculate luminance value for.

The type of reflection from the roadway surface and thus the reflection characteristics of the tarmac plays a key role in the sensitivity of the lighting scenario to the modification of some key light intensity values of the light distribution ${ }^{[5]}$. By the investigations the three most common road types have been evaluated; $\mathrm{R} 1, \mathrm{R} 2$, and $\mathrm{R} 3$ reflection classes.

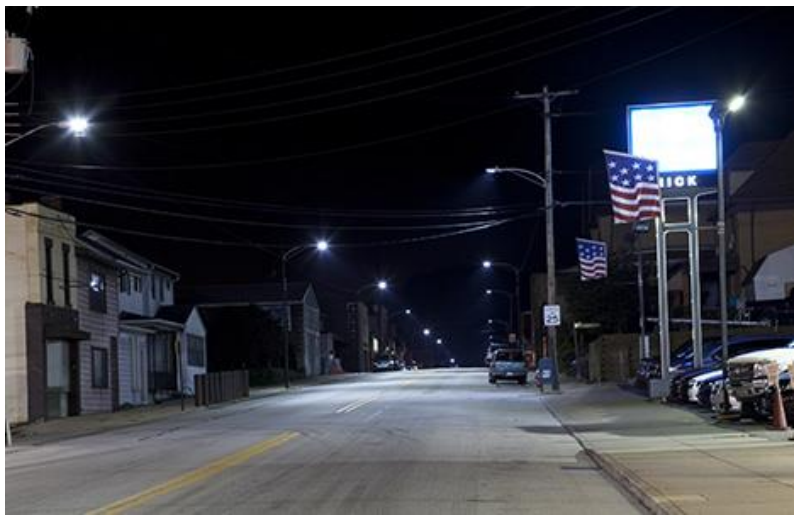

${ }^{\text {d. }}$ A typical perceived image of a well-lit roadway; GE Lighting, Evolve LED fixtures

Image (d) shows a typical scene of roadway lighting. While there are identical SKUs in a staggered layout over the roadway, that emit the highest light intensity towards the nadir section (downwards), the roadway seems brighter beneath the luminaire only for the closest poles, and farther there is even a darker spot observable around the same relative location. This is due to the specular dominant reflection characteristics of the given roadway that works as a mirror in this situation. If the output light distribution was modified here by an amount of $10 \%$ in terms of light intensity - more light to the nadir, less to the corner section - the scene would seem more uniform farther and might be even disturbing at the closer surfaces. The lighting design shown on this image is excellent, because it provides excellent contrast of obtrusions over the roadway.

\section{Uniformities}

The uniformity of Illuminance and Luminance are key esthetical parameters of a lighting design. Usually the ratio of minimum to average or minimum to maximum is specified. Excessive values of both parameters result in disturbing lighting, glare and decreased contrast perception ${ }^{[4]}$.

\section{Threshold Increment (TI)}

Threshold increment is the measure of disability glare expressed as the percentage increase in contrast required between an object and its background for it to be seen equally well with a source of glare present. Its value is expressed in percentage, the higher it is, the more glare is caused by the installation [6]

TI always rates the installation and not the luminaire light distribution, as it is the ratio of the average Luminance perceived to the peak Illuminance cast on the observer.

While by the standards the defined maximum value is given, for LED applications the authorities preparing the tender requirements often challenge the lighting designers a stricter maximum value. The main reason is that LED light sources have a higher task efficiency that results a different adaptation process than what is justified for core lighting products, such as HPS and CMH lamps.

\section{OPTICAL PERFORMANCE SHIFTING OF DIFFERENT DESIGNS}

In the previous section the key parameters have been introduced, that measure the performance of a luminaire in a given installation. For a brief comparison, these indicators were compared on a sample scenario for three different mounting methods over a specified ambient temperature range.

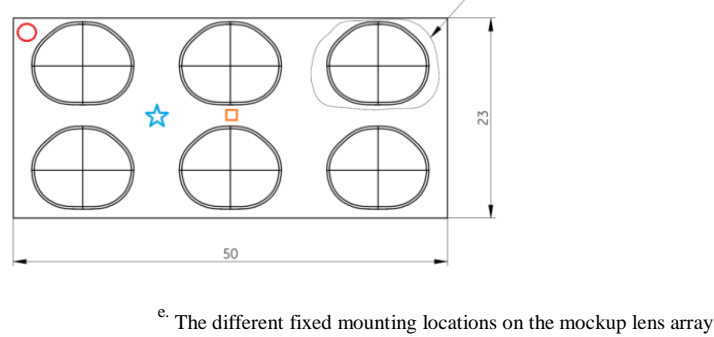

Image (e) shows a sample lens array containing six lens in a cluster of two by three. Ideally at rated ambient temperature the LEDs center point is at the cross in the center of the lens. 
There are three marks on the drawing, a red circle, an orange box and a blue star. There specify the location of the fix mounting position, that holds its relative position to the board during thermal expansion. The dimensions indicated are in $\mathrm{mm}$.

For the evaluation of these distributions an optical ray tracing simulation was made and the resulting light distributions have been analyzed in a lighting design software at different temperatures.

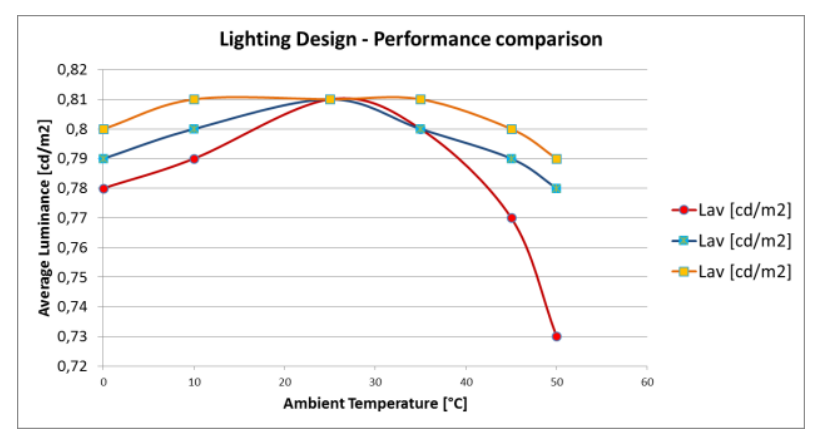

${ }^{\text {f. }}$ Lighting design, performance comparison of average Luminance

Diagram (f) shows the results of the lighting design calculations highlighting the average luminance achievable at different ambient temperatures. It is clear to see, that the optics performs best at the rated ambient temperature, while becomes less competitive outside the optimal conditions.

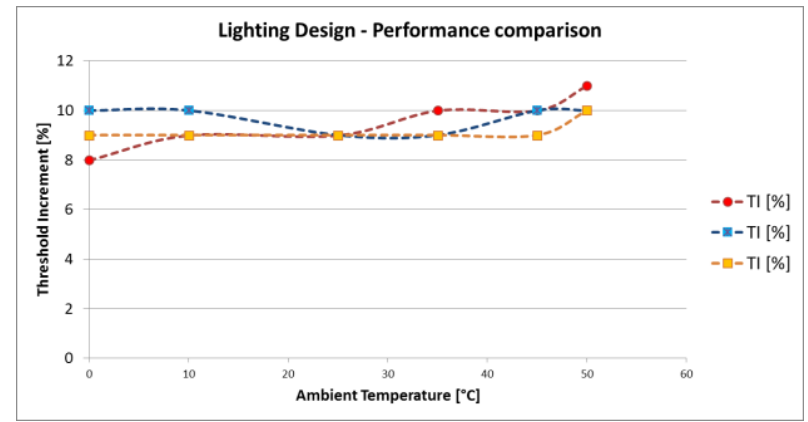

g. Lighting design, performance comparison of Threshold Increment

Diagram (g) shows the results for Threshold Increment. The fact that this is not linear on any of the parameters (ambient temperature and mounting) represents the complexity of this performance indicator ${ }^{[7]}$.

\section{SUMMARY}

It was shown, that the temperature during operation of any given part can vary on an excessive range in an outdoor lighting LED luminaire. Due to the fact that the thermal expansion coefficient also varies between the mated components, the relative positions, especially for the optical light engine are suffering a serious shifting compared to the original design position at the rated ambient temperature.

A brief evaluation has been introduced in order to visualize the effect of this shifting on real life lighting design scenarios. While a refractive lens based optical system may perform outstandingly in a given environment, it is possible, that changing the ambient temperature, humidity or even air flow properties, the same product can become inadequate by failing the lighting requirements.

\section{References}

[1] Duco Schreuder - Outdoor Lighting: Physics, Vision and Perception, 2010 Springer Science, ISBN: 978-90-481-7930-5.

[2] Peter R. Boyce - Lighting for Driving, CRC Press, 2009, ISBN-13: 978-0-8493-8529-2

[3] Kohei Narisada, Duco Schreuder - Light Pollution Handbook, 2004 Springer Science, ISBN: 978-1-4020-2666-9 (e-Book).

[4] NÉMETH Z, NAGY B V, ÁBRAHÁM Gy, VERES A, SAMU K HOW TO CHOOSE SIMULATION PARAMETERS TO IMPROVE ACCURACY?, Proceedings of CIE Centenary Conference: Towards a New Century of Light. 2013.04.15-2013.04.16.

[5] http://eilv.cie.co.at/term/1312 - Referenced: 2015.05.25.

[6] Németh Zoltán, Veres Ádám, Ábrahám György, Samu Krisztián Development of outdoor luminary using SPEOS software, LUMEN V4 IV. Lighting Conference of the Visegrad Countries. 2012.09.262012.09.28. 